\title{
Role of suction blistering in treatment of patients with stable vitiligo
}

\author{
Sara H. Ahmed, Samia M. Ahmed, Reham E. Elsharkawy,Mohammed A. Ali
}

\begin{abstract}
Introduction:Vitiligo is a common depigmenting disorder, characterized by milky white macules due to absence of functional melanocytes in the affected area. Vitiligo is the most prevalent pigmentary disorder with an incidence rate between $0.1-2 \%$ showing multifactorial etiology and polygenic inheritance.
\end{abstract}

Aim of the work:To study the efficacy and side effects of suction blister in treatment of patients with stable vitiligo.

Patients and Methods:Ten patients with stable vitiligo attending dermatological outpatient clinic at Sohag university hospital were treated with suction blistertechnique.

Results:Suction blistering is an easy and effective technique in treating stable vitiligo.

Key words:Stable vitiligo- Suction blister- Repigmentation- Quality of life (QoL).

\section{Introduction}

Vitiligo is a common depigmenting disorder, characterized clinically by milky white macules and histologically by an absence of functional melanocytes in the affected area. It causes severe cosmetic distress, particularly in darkly pigmented skins and is also associated with a great social stigma(Alkhateeb et al., 2003).There are many potential pathophysiological theories involving autoimmune, neural, autocytotoxic, biochemical, oxidative stress, melanocytorrhagy, and decreased melanocyte survival hypotheses(Spritz, 2006).

Vitiligo has a significant impact on QoL, affecting activities of daily living and personal relationships. Psychological counseling and support is necessary in the management of vitiligo(Al-Harbi, 2013).

The goal of vitiligo treatment is to control the autoimmune damage to melanocytes and stimulate their migration from surrounding skin and adnexal reservoirs. The medical treatment of vitiligo is effective in only 60$70 \%$ of the patients. Certain types of vitiligo do not respond well to medical treatment and resistant lesions do persist even in those who respond. In light of these limitations of medical treatment, surgical treatment of vitiligo was first proposed in the 1960s
(Parsad and Gupta, 2008; Donaparthi and Chopra, 2016). These surgical therapies, as a rule, are indicated in those patients who have a stable (non-progressive) disease of at least one year and not responding to medical treatment(Parsad and Gupta, 2008).

Suction blister grafting (SBG) is a technique where the pigmented epidermis is harvested from the donor site by using suction to raise a blister which is then transferred to the vitiliginous area (Rusfianti and Wirohadidjodjo, 2006).

Patients and Methods

A prospective clinical study was performed on 10 patients with stable vitiligo attending dermatological outpatient clinic at Sohag university hospital. The study was submitted for approval by Research and Ethical committee at Faculty of Medicine, Sohag University. Written consent was obtained from all patients.

All the patients were subjected to complete history including (age, sex, duration of vitiligo, duration of stability, previously received treatment and systemic diseases). Then complete examination was conducted (general and dermatological examination).

Technique of suction blisterimg: 
The donor site was selected on the medial aspect of the arm. After local anesthesia, Both donor and recipient sites were attached to base of syringe $20 \mathrm{~mm}$ which was connected to IV line device connected to syringe $50 \mathrm{~mm}$ to produce negative pressure. After about 3-4 hours of application of suction, the blister was ready and was removed by scissor and placed in a dish containing normal saline. Then the donor site was dressed with antibiotic ointment and Vaseline gauze.

After removing the roof of the donor and recipient site blister, donor graftable epidermis was placed on the recipient site, then covered with antibiotic ointment and Vaseline gauze with the dressing firmly bound in place with a compression bandage, and a 7-day course of antibiotic was given. The dressing was removed after 2 weeks. Photographs were taken before and after procedure.

Follow up was done every 2 weeks for 3 months with photographic assessment and all patients were subjected to phototherapy with narrow band UVB at $311 \pm 2 \mathrm{~nm}$ wavelength (Waldmann UV 100L, Germany).

The response to the treatment based on the percentage of visible repigmentation was graded as follows: Poor: $0-25 \%$; good: $26-50 \%$; very good: $51-75 \%$; and excellent: $76-100 \%$ and quality of life questionnaire before and after the study.
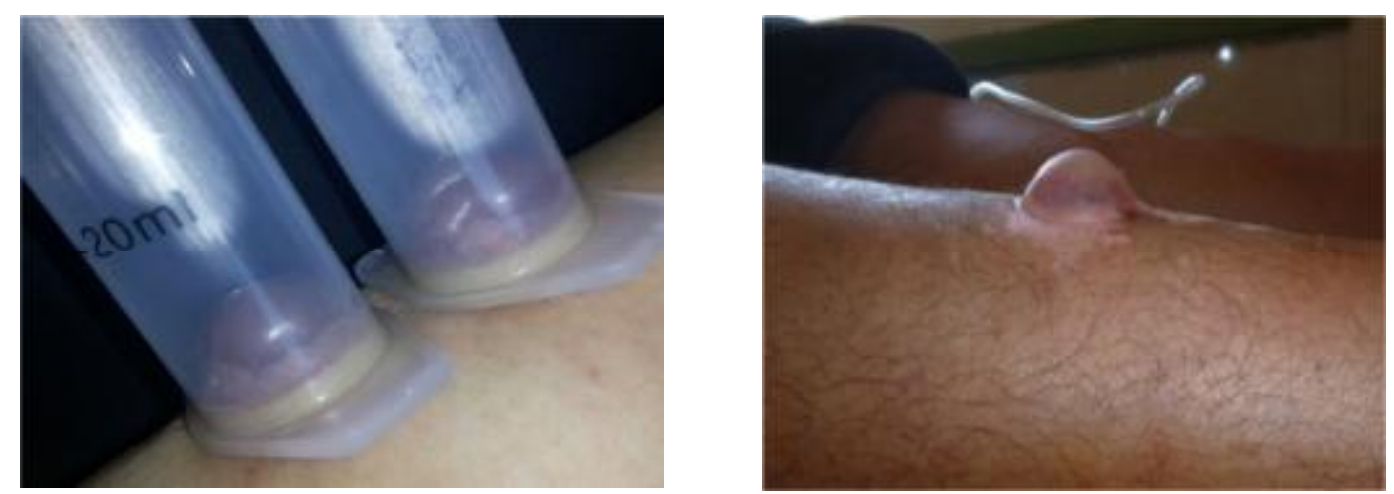

Figure (1): Suction blistering techniqueFigure (2) Suction Blister formation

ResultsTable (1): characteristics of the studied population.

\begin{tabular}{|l|c|}
\hline Variable & Suction blister technique $(\mathbf{n}=10)$ \\
\hline Age/year & $27.2 \pm 15.20$ \\
Mean \pm SD & $27(\mathbf{1 6 - 6 0 )}$ \\
Median (range) & $\mathbf{8 ( 8 0 . 0 0 \% )}$ \\
\hline Gender & $2(20.00 \%)$ \\
Females & $7(70.0 \%)$ \\
Males & $\mathbf{3 ( 3 0 . 0 0 \% )}$ \\
\hline Family history & \\
Negative & $2.1 \pm 1.20$ \\
Positive & $2.1(1-5)$ \\
\hline Duration of stability & \\
Mean \pm SD & \\
Median (range) & \\
\hline
\end{tabular}


Table (2):Repigmentation effect of suction blistering.

\begin{tabular}{|l|c|}
\hline Variable & Suction technique \\
\hline $\begin{array}{l}\text { Percent of repigmentation } \\
\text { Mean } \pm \text { SD }\end{array}$ & $90 \pm \mathbf{1 0 . 5 4}$ \\
Median (range) & $90(\mathbf{7 0 - 1 0 0 )}$ \\
\hline Repigmentationcategories & \\
Excellent & $9(90.0 \%)$ \\
Very good & $1(\mathbf{1 0 . 0 \%})$ \\
Good & 0 \\
Poor & 0 \\
\hline
\end{tabular}

Table (3):Complications of suction blistering.

\begin{tabular}{|l|ll|}
\hline Complications at recipient site & Complicationsat donor site \\
\hline Hyperpigmentation $1(\mathbf{1 0} \%)$ & Hyperpigmentation & $\mathbf{2}(\mathbf{2 0 \%})$ \\
\hline Cobblestonning $\quad \mathbf{0}$ & \multirow{2}{*}{ Cobblestonning } & $\mathbf{0}$ \\
\hline Rejection of graft $\mathbf{0}$ & &
\end{tabular}

Table (4):Quality of life (QOL) score of suction blistering.

\begin{tabular}{|l|c|}
\hline Variable & Suction technique \\
\hline QOL score before operation & \\
Mean \pm SD & $18.5 \pm 1.51$ \\
Median (range) & $18.5(16-21)$ \\
\hline QOL score after operation & \\
Mean \pm SD & $1.6 \pm 2.07$ \\
Median (range) & $\mathbf{1 . 6}(0-7)$ \\
\hline P compared before and after & $<0.0001$ \\
\hline
\end{tabular}
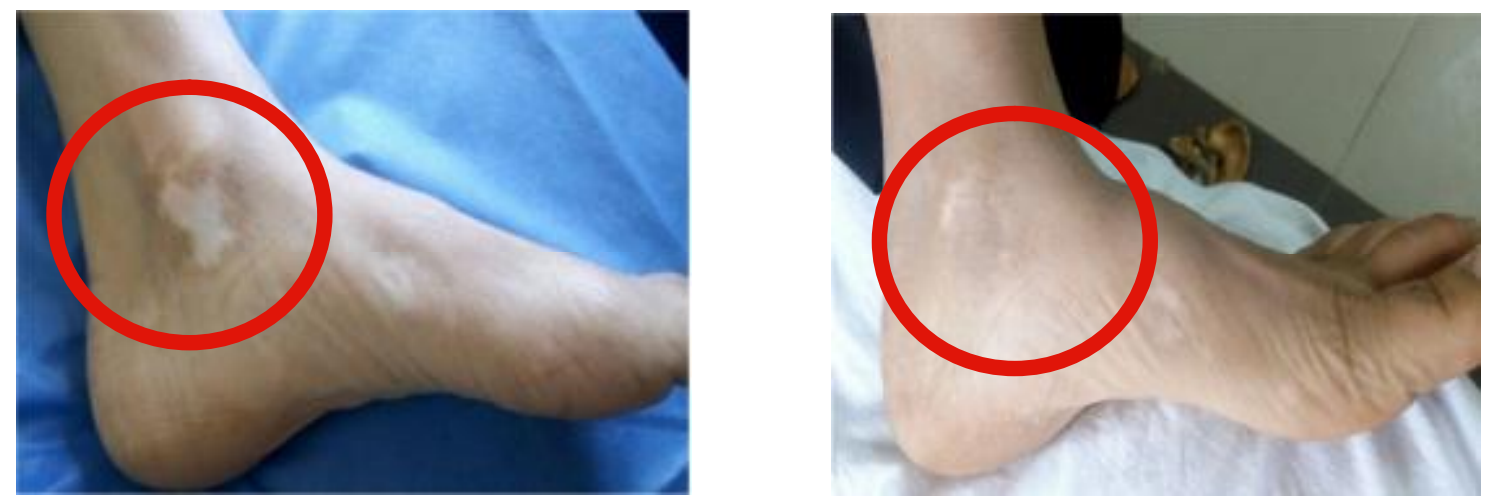

A)

B) 
Figure (3): A) Preoperative. B) Postoperative 3 months after suction blistering

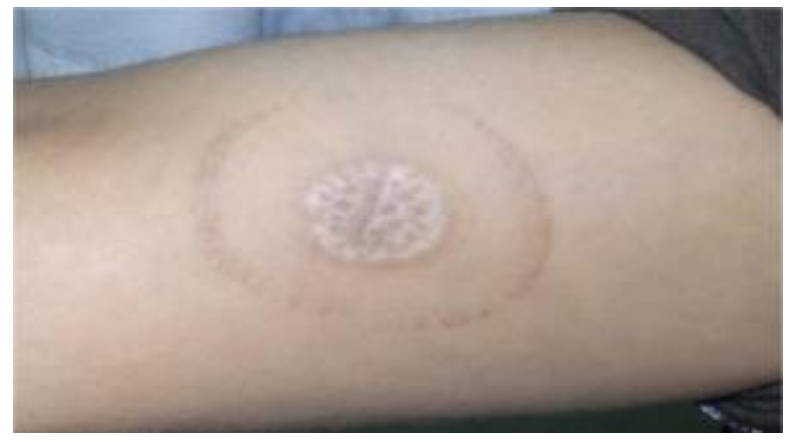

Figure (4): Hyperpigmentation at donor site

\section{Discussion}

Vitiligo is a chronic disfiguring disease of unknown origin that causes destruction of melanocytes in the skin, mucous membrane, eyes, inner ear and occasionally hair bulbs. It has an unpredictable clinical course(Sharma et al., 2004).

In this study, suction blistering had high percentage of repigmentation $(90 \pm 10.54)$. The mean onset of repigmentation in suction blistering was (3.5 \pm 0.53$)$ weeks.Gupta et al in 1999 recorded that the repigmentation on vitiligo patients were $(82 \%)$ patches in suction blistering epidermal graft (SBEG).

Maleki et al., 2012 found that blister grafting without phototherapy showed excellent results in $70 \%$ of patients. The likely explanation why suction blistering gave the best results is that the graft is superficial and cleavage occurs between the basal cells and the basal lamina of the basement membrane zone and only the epidermal portion of the donor area is grafted, thus leading to a better color match and cosmetic outcome (Khunger et al., 2009).

In this study, the suction blistering technique had the least rate of complications; only $10 \%$ had hyperpigmentation at donor site.Babu et al 2008 reported thathyperpigmentation and thickening of grafts were common in suction blistering technique.

Suction blistering technique is easy, simple method with good cosmetically results with low cost, absence of scarring and the possibility of reusing the donor site, On the contrary it is time consuming, painful and not suitable for large areas, uneven surfaces and the palm (Maleki et al., 2012).

\section{Summary}

Vitiligo is a chronic disfiguring disease that affects approximately $0.5 \%$ to $2 \%$ of the population worldwide. The medical treatment of vitiligo is effective in only $60-70 \%$ of the patients. Surgical treatment of vitiligo is an effective and alternative method for treatment of stable vitiligo resistant to medical treatment. Suction blistering technique is easy, simple method with good cosmetically results and less side effects.

\section{References}

1. Al-Harbi M: Prevalence of depression in vitiligo patients. Skinmed, 2013; 11, 327-30.

2. Alkhateeb A, Fain PR, Thody A, Bennett DC and Spritz RA: Epidemiology of vitiligo and associated autoimmune diseases in Caucasian probands and their families. Pigment Cell Res14-208، 16 2003 ،

3. Babu A, Thappa DM, Jaisankar TJ:Punch grafting versus suction blister epidermal grafting in the treatment of stable lip vitiligo. DermatolSurg, 2007; 34, 166-78.

4. Donaparthi $\mathbf{N}$ and Chopra A: Comparative Study of Efficacy of Epidermal Melanocyte Transfer versus Hair Follicular Melanocyte Transfer in Stable Vitiligo. Indian J Dermatol, 2016; 61, 640-4.

5. Gupta S, Jain VK and Saraswat PK: Suction blister epidermal grafting versus 
punch skin grafting in recalcitrant and stable vitiligo. Dermatol Surg, 1999; 25, 955-8.

6. Khunger N, Kathuria SD and Ramesh V: Tissue grafts in vitiligo surgery - past, present, and future. Indian $J$ Dermatol, 2009; 54, 150-8.

7. Maleki M, Banihashemi M, Sanjari V: Efficacy of suction blister epidermal graft without phototherapy for locally stable and resistant vitiligo. Indian J Dermatol, 2012; 57, 282-4.

8. Parsad D and Gupta S: Standard guidelines of care for vitiligo surgery.
Indian J Dermatol Venereol Leprol, 2008; 74 Suppl, S37-45.

9. Rusfianti Mand Wirohadidjodjo YW: Dermatosurgical techniques for repigmentation of vitiligo. Int $J$ Dermatol, 2006; 45, 411-7.

10. Sharma L, Bhawan $R$ and Jain RK: Hypoacusis in vitiligo. Indian $\mathrm{J}$ Dermatol Venereol Leprol, 2004; 70, 162-4.

11. Spritz RA: The genetics of generalized vitiligo and associated autoimmune diseases. J Dermatol Sci, 2006; 41, 3-10. 